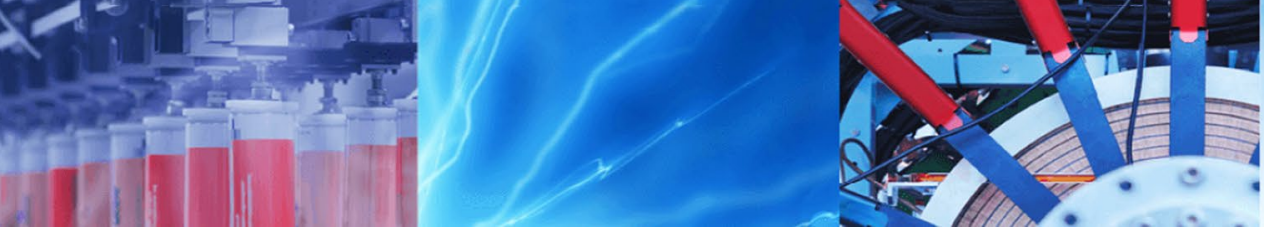

Research Article

\title{
Classification of lymphocytes, monocytes, eosinophils, and neutrophils on white blood cells using hybrid Alexnet-GoogleNet-SVM
}

\author{
Ahmet Çınar ${ }^{1} \cdot$ Seda Arslan Tuncer ${ }^{2}$
}

Received: 20 November 2020 / Accepted: 9 March 2021 / Published online: 25 March 2021

(C) The Author(s) 2021 OPEN

\begin{abstract}
White blood cells (WBC), which form the basis of the immune system, protect the body from foreign invaders and infectious diseases. While the number and structural features of WBCs can provide important information about the health of people, the ratio of the subtypes of these cells and observable deformations are a good indicator in the diagnostic process. The recognition of cells of the type of lymphocytes, neutrophils, eosinophils, basophils and monocytes is critical. In this article, Deep Learning based Hybrid CNN (Convololutional Neural Network) model is proposed for classification of eosinophils, lymphocytes, monocytes, and neutrophils WBCs. The model presented is based on pretrained Alexnet and Googlenet architectures. The feature vector in the last pooling layer of both CNN architectures has been merged, and the resulting feature vector is classified by the Support Vector Machine. To determine the superiority of the proposed method, the classification was also performed and compared using pretrained Alexnet and Googlenet. Hybrid AlexnetGooglenet-SVM model provides higher accuracy than pretrained Alexnet and Googlenet. The proposed method has been tested with WBC images from Kaggle and LISC database. Accuracy and F1-score were 99.73\%, 0.99 and 98.23\%, 0.98 for both data sets, respectively.
\end{abstract}

Keywords WBC classification · Hybrid CNN · Alexnet-Googlenet-SVM

\section{Introduction}

White blood cells are important building blocks of the immune system that help fight infection and protect the body against foreign substances such as viruses. Diagnosis of diseases such as blood cancer, AIDS from WBCs is important for hematologists. Separating WBC cells into subtypes is difficult due to differences in cell shape in images during maturation. To overcome this problem, machine learning and new generation CNN structures have been proposed [1-5]. Macawile et. al proposed a method that can segment cells from microscopic blood images. The proposed method is based on CNN, which can classify, monocytes, neutrophils, lymphocytes, basophils and eosinophils from a microscopic blood image of Hue Saturation Value [6]. Sahlol et al. proposed an advanced hybrid approach to the effective classification of Leukemia. Firstly, features were extracted from WBC images using VGGNet. Secondly, the obtained features are filtered with the Salp Swarm Algorithm. The proposed approach has been applied to two general WBC reference datasets. Accuracy and reduced computational complexity were achieved according to the obtained results. [7]. Ramesh et al. proposed a classify framework based on color information and morphology. The performance of the algorithm was evaluated by comparing the visual classification of the hematopatologist. The algorithm was applied to the 1938 sub-image of WBCs, of which 1804 were correctly classified. Later, in the

$\checkmark$ Seda Arslan Tuncer, satuncer@firat.edu.tr; Ahmet Çınar, acinar@firat.edu.tr | ${ }^{1}$ Faculty of Engineering, Computer Engineering, Fırat University, Elazığ, Turkey. ${ }^{2}$ Faculty of Engineering, Fırat University, Software Engineering, Elazı̆̆, Turkey. 
two-stage classification, WBCs were classified into cells with broadly segmented nuclei and non-segmented nuclei. In the second stage, Feature description has been made for classifying WBCs by linear discriminant analysis. System evaluation was made using $\mathrm{k}$-fold cross validation technique. An overall accuracy of $93.9 \%$ was determined in the five subtype classifications of the applied two-stage classification [8]. Su et al. proposed a new algorithm for segmentation of WBCs from smear images. The main idea of the proposed algorithm is to find a distinctive region of WBCs in the HSI (Hue Saturation Intensity) color space. Three types of properties (i.e. geometric properties, color properties and LDP-based tissue properties) have been extracted and given to three different neural networks to recognize the types of WBCs. A total of 450 WBC images were used to test the effectiveness of the proposed WBC classification system. The highest accuracy rate was 99.11\% [9]. Kutlu et al. in his studies, blood cells were classified with Regional Based Evolutionary Neural Networks. The proposed architectures have been trained and tested by combining the BCCD and the LISC (Leukocyte Images for Segmentation and Classification) data set. Classification was implemented by using AlexNet, VGG16, GoogLeNet, ResNet50 architectures. the proposed system showed $100 \%$ success in identifying WBCs. Lymphocyte cell types were determined with $99.52 \%$ accuracy rate, $98.40 \%$ accuracy rate, Monocyte, $98.48 \%$ accuracy with Basophil, 96.16\% accuracy with Eosinophil and $95.04 \%$ accuracy with Neutrophil in Resnet50 architecture[10]. Barrero et al. developed a system to classify and identify blood cells using networks of Gauss Radial Base Functions (RBFN). While it is generally $97.9 \%$ accurate in the classification of WBCs, the sensitivity in classification by cell type is $93.4 \%$ for lymphocytes, $79.5 \%$ for neutrophils, $97.37 \%$ for monocytes, $73.07 \%$ for eosinophils and $100 \%$ for basophils according to professionals [11]. Habibzadeh et. at. examined the classification of WBCs according to four major types, including Eosinophils, Neutrophils, Lymphocytes and Monocytes, using the Deep Learning. After the preprocessing phase, WBC recognition was realized with hierarchy topological feature extraction by means of ResNet and Inception architectures. For training and testing were used 11,200 and 1244 images respectively. ResNet50 detected an average of $100 \%$ of the four main WBC types, while promising results were obtained with the accuracy rate of $99.84 \%$ and $99.46 \%$ obtained with ResNet 152 and ResNet101. Other statistical confusion matrix tests revealed that this study reached precision values of 1.0, $0.9979,0.9989$ if the Area Under the Curve (AUC) exceeds $1.0,0.9992,0.9833$ in the three proposed techniques [12]. Zhao et al. proposed an automatic detection and classification system for WBCs from blood images. First of all, an algorithm was developed to detect WBCs from microscope images based on their simple relationship with the morphological study of R, B colors. Next, a granularity feature (Bidirectional Invariant Local Formation Local Pattern, Pairwise rotation invariant co-occurrence local binary pattern feature) and SVM (Support Vector Machine) were first applied to classify eosinophils and basophils from other WBCs. Finally, CNNs are used to automatically extract highlevel features from WBCs, and random forest algorithm was applied to obtained features to recognize the type of WBCs. Experiments on the ALL-IDB(Acute Lymphoblastic Leukemia Image Database for Image Processing) and Cellavison database have been shown to have a better effect than the iterative threshold method of the proposed method [13]. Ruberto et al. proposed a new method to recognize WBCs from microscopic blood images. Images are classified as healthy or influenced by leukemia. The proposed system has been tested in general data sets for leukemia detection such as SMC-IDB, IUMS-IDB databases. The results were promising, but $100 \%$ accuracy for the first two data sets and $99.7 \%$ for ALL-IDB in detection of white cells and $94.1 \%$ in leukemia classification [14]. Baydili et. al classified WBC images into five categories through capsule networks, a new method of deep learning. The results obtained with the model were compared with the most known deep learning methods and a high accuracy was obtained in the test data (96.86\%) [15]. Gupta et al. proposed the Optimized Binary Bat Algorithm (OBBA) for the classification of different types of leukocytes. An optimized algorithm is used to obtain a subset of these features by removing a number of features from the images of the WBCs. The proposed algorithm was implemented using four different classifiers using the k-NN (k-Nearest Neighbor), Logistic Regression, Random Forest, and Decision Tree, and their performance was compared. The proposed OBBA classifies WBCs with an average sensitivity of $97.3 \%$ [16]. Shahin et al. proposed a new identification system for WBCs based on CNN. In addition, a new end-to-end evolutionary deep architecture called "WBCsNet" has been developed. As a result of tests performed on three different general WBC datasets (2551 images), accuracy of $96.1 \%$ was obtained with the proposed WBCsNet [17]. Togaçar et al. focused on classifying WBC images using the CNN models. Various classifiers have been used on properties derived from AlexNet architecture to evaluate classification performance. The best performance was obtained by the Quadratic Discriminant Analysis classifier with an accuracy of $97.78 \%$ [18]. Hedge et al. proposed a classifier that can detect abnormal cells as well as white blood cells. In the study, traditional image processing approach and deep learning methods were compared for classification of WBCs. An accuracy of around $99 \%$ was achieved for CNN [19]. Malkawi et al. using a hybrid system using CNN and different machine learning algorithms (SVM, KNN and 
Random Forest), they classified WBC cells with $98.7 \%$ accuracy[20]. Rezatofighi et al. proposed image processing algorithms to automatically recognize WBC cells. Using a two-step process, the cell nucleus and cytoplasm were segmented primarily based on Gram-Schmidt orthogonalization. Then, various features were extracted from the segmented regions, and the classification was performed with the Artificial Neural Network and SVM [21]. Pinyakupt et al. performed both segmentation and classification of five types of WBC cells using linear and Naive Bayes algorithms. The proposed system consists of preprocessing step, nuclei segmentation, cell segmentation, feature extraction, feature selection and classification processes. The accuracy obtained with Linear and Naive Bayes classifiers was $98 \%$ and $94 \%$ respectively [22]. Sarrafzadah et al. used extracted features from both the nucleus and the cytoplasm, in addition to the properties doctors used when classifying WBC cells. In the method using SVM algorithm, the classification was achieved with $93 \%$ accuracy [23].

Algudah et al. extracted three distinct properties from WBCs: morphological, statistical, and textural. Principal component analysis (PCA) was used to determine the order of the extracted features. Classification was carried out with probabilistic neural network (PNN) and support vector machine and random Forest Tree. The accuracy obtained was $99.6 \%$ [24].

Today, in the age of modernization, there is a great deal of research in the field of image processing combined with various segmentation and classification techniques to produce alternatives for WBC classification and counting. In these studies, the previous identification systems for the classification of WBCs consist of preprocessing, partitioning, feature extraction, and feature selection steps. The accuracy of these existing methods is still improved. It is a real need to use deep learning methodologies to improve the performance of the identification systems of previous WBCs.

The purpose of this article is to develop a system for the identification and classification of WBCs, using image processing techniques to support the doctor in the diagnostic process, and to reduce the subjective errors in manual analysis. Therefore, we proposed the
Alexnet-Googlenet-SVM hybrid CNN method, which can classify various types of WBC. The proposed method includes pre-processing, filtering, feature extraction and classification processes as a whole. The system uses Alexnet and Googlenet architectures, which are pretrained models. WBC image feature vectors in the last pooling layer of these models were combined and classification was carried out with the help of SVM algorithm.

The contribution of paper is as follows:

- Using 2 different data sets, Eosinophil, Lymphocyte, Monocyte and Neutrophil WBC images were classified by Alexnet-Googlenet-SVM hybrid CNN method.

- The new feature vector was obtained by concatination by taking the maximum values of Alexnet and Googlenet's feature vectors.

- The feature vectors obtained were classified with SVM.

- Considering both the literature and the results obtained, WBC classification was performed with high accuracy with the Hybrid CNN model.

The rest of the article is organized as follows. Data and properties used for classification are presented in Sect. 2. The structure of the method proposed in Sect. 3 is detailed. The results and discussion obtained in Sect. 4 are presented. In the last section, a brief evaluation of the proposed method is given.

\section{Data sets}

In this article, two different data sets from the Kaggle website and LISC database are classified $[25,26]$. The data set from the Kaggle website contains 12,500 enhanced blood cell JPEG images with cell-type tags (CSV). There are approximately 3000 images for each of the 4 different cell types grouped in 4 different folders. Cell types are Eosinophil, Lymphocyte, Monocyte and Neutrophil. For each class, 248 were used for a total of 992 image tests. Figure 1 shows an example image for each class.

In the LISC dataset, Samples have been taken from peripheral blood of 8 normal subjects and 400 samples have been obtained from 100 microscope slides. these

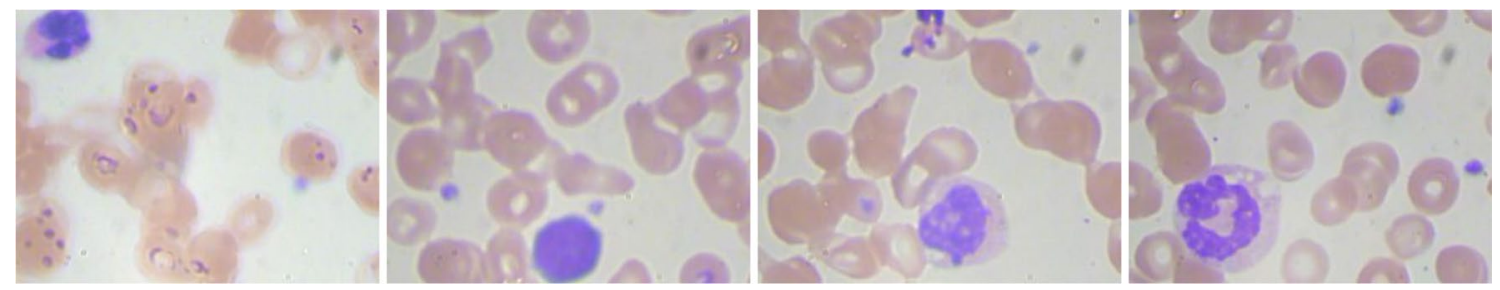

Fig. 1 Images of eosinophil, lymphocyte, monocyte and neutrophil, respectively 
images have been recorded by a digital camera and have been saved in the BMP format. The size of the images is $720 \times 576$ pixels.

Color images have been collected from hematologyOncology and BMT Research Center of Imam Khomeini hospital in Tehran, Iran. The images were classified by a hematologist into normal leukocytes: eosinophil, basophil, monocyte, lymphocyte, and neutrophil. In this article, a total of 189 eosinophil, lymphocyte, monocyte, and neutrophil WBCs images were used for classification. By applying augmentation to these images, the number of data was 99 for each class. The applied augmentation method is rotation. Images were subjected to 90,180 and 270 degrees of rotation.

\section{The proposed methot}

Convolutional Neural Networks (CNN) such as the first developed Alexnet [27] and Googlenet[28] have been successfully applied to problems such as medical classification [29-31]. Alexnet and Googlenet based hybrid CNN model, which are pretrained models for classification of WBC images, are as in Fig. 2. The model takes WBC images as input. Convolution, normalization and pooling layers are applied on each image. Thanks to these steps, feature vectors of each image are obtained. These feature vectors obtained in pretrained models are classified by means of softmax layer. In the proposed model, a single feature vector was obtained by combining feature vectors in the last pooling layers of Pretrained models. The resulting feature vector is classified by SVM algorithm instead of softmax. The proposed hybrid Alexnet-Googlenet-SVM model base layer properties are described below.
Convolution: This layer, which is responsible for perceiving the properties of the image, is the main structure of CNN. It is applied to eliminate some features that do not need to be trained in images. In the Convolution layer, $\mathrm{k}$ filtering is applied to $W \times Y \times D$ size data, where $k$ is filter size, $W, Y$, and $D$ are width, heigth and depth of input image. The width and depth of the new data to be obtained by applying the filter are calculated according to Eqs. 1 and 2.

Output Width $=\left(\frac{W-F_{w}+2 P}{S_{w}}\right)+1$

Output Height $=\left(\frac{W-F_{h}+2 P}{S_{h}}\right)+1$

where, $F h, F w, S w, S h$ and $p$ show Filter height and width, Stride width and height, Padding respectively.

Activation Layer: This layer, which comes after all Convolutional layers, is used to remove the linearity in the image. In this layer, the Rectifier (ReLu) function is often used because of its speed advantage (Eq. 3).

Relu : $f(x)=\left\{\begin{array}{l}0, x<0 \\ x, x \geq 0\end{array}, f(x)^{\prime}=\left\{\begin{array}{l}0, x<0 \\ 1, x \geq 0\end{array}\right.\right.$

Pooling layer: This layer is a layer that is frequently added between convolutional layers. The task of this layer is to reduce the shift size in the image and the parameters and calculations in the network. There are many pooling operations used in the literature such as average pooling, max pooling and L2-norm pooling. Equation 4 is used to obtain the properties in this layer.

$O M=\left(\frac{I M+2 P-F}{S}\right)+1$

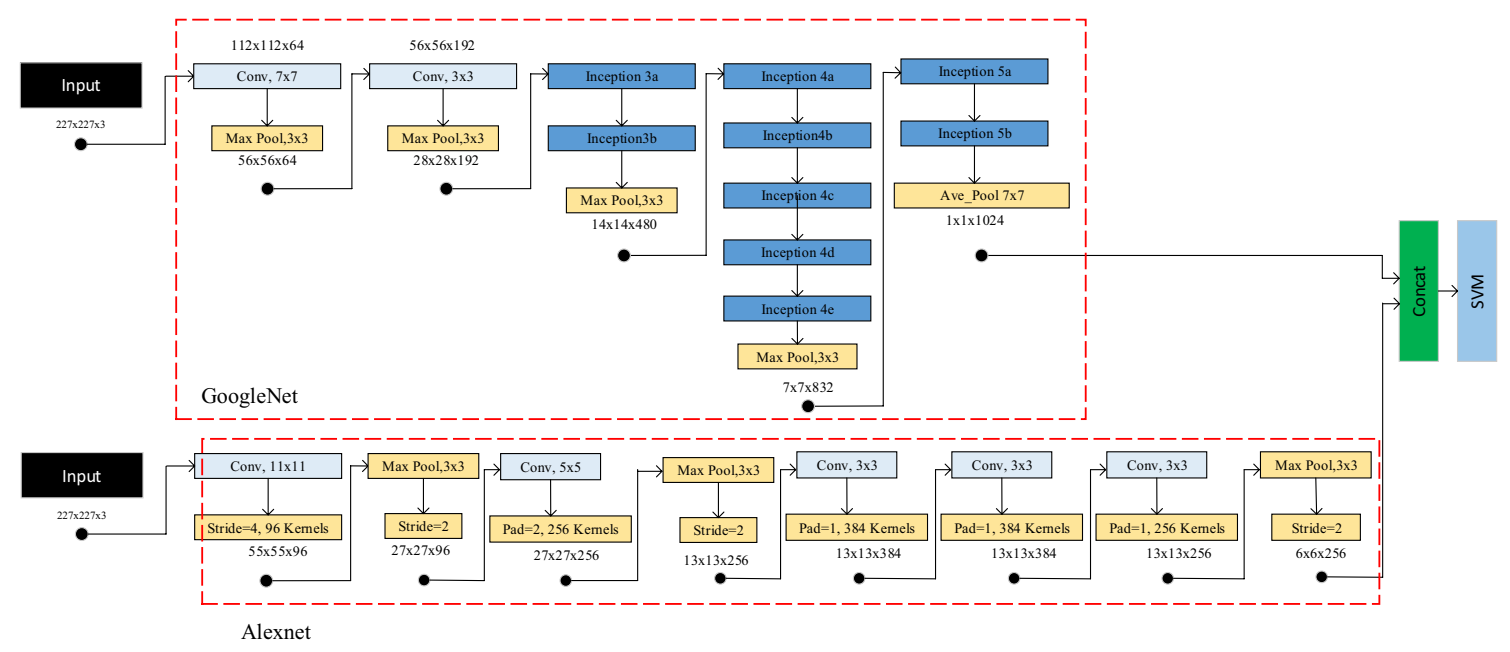

Fig. 2 The proposed hybrid Alexnet-Googlenet-SVM model 
where, $I M, O M$ and $S$ are Input Matrix, Output Matrix and Stride, respectively.

Normalization: Normalizes the output produced by the convolution and fully connected layers to improve the training time of the network. Equation 5 shows the normalization equation.

$Y_{i}=\frac{X i-\mu_{\beta}}{\sqrt{\sigma_{\beta}^{2}+\varepsilon}}$

Depending on Eq. 5, $\sigma_{\beta}$ and $\mu_{\beta}$ are calculated as in Eqs. 6 and 7.

$\sigma_{\beta}=\frac{1}{M} \sum_{i=1}^{M}\left(X_{i}-\mu_{\beta}\right)^{2}$

$\mu_{\beta}=\frac{1}{M} \sum_{i=1}^{M} X_{i}$

where $M, \mu_{\beta}, \sigma_{\beta}$ and $Y_{i}$ are respectively, number of input data, average ans standard deviation of the stack, new values resulting from normalization process.

Inception module: Each module consists of different sized convolution and max-pooling processes. The expansion effect in the Inception modules is created by parallel execution of $1 \times 1,3 \times 3,5 \times 5$ filters and $3 \times 3$ maximum sharing in the convolution layers. The purpose of this layer is to optimize the processing load. Figure 3 Inception $3 a$ shows the internal architecture of the module.

Concat: The feature vectors of the Pool5 and Average Pool layers of the Alexnet and Googlenet architectures used in the proposed model are 9216 and 1024 for each image. This layer performs the merge of feature vectors. To eliminate the size difference between the two feature vectors, the padding process is applied to the feature vector in the Googlenet Average_Pool layer and the size is equalized to the Alexnet feature vector. Thus, a single feature vector was obtained by combining two different feature vectors of a single image. The feature vector representing the image from these feature vectors was obtained

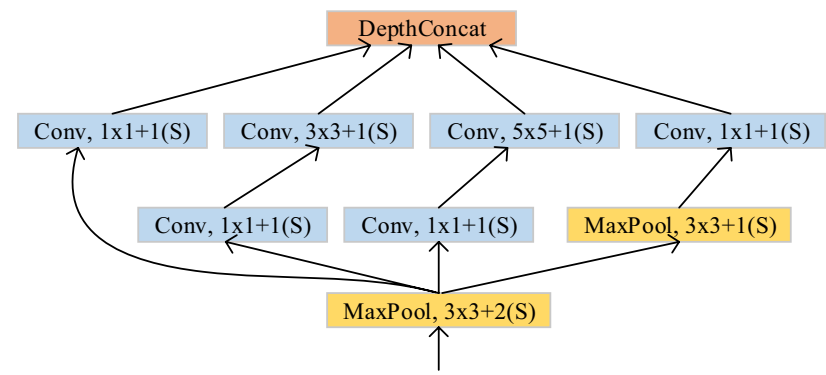

Fig. 3 Inception 3a Module architecture by applying maximization. Figure 4 shows the application of the maximization process to the Alexnet and Googlnet feature vectors.

\section{Experimental results and discussion}

A computer with Intel core $\mathrm{i} 7-9750 \mathrm{H}$ processor and $8 \mathrm{~GB}$ RAM was used in the tests. When using Transfer Learning for Pretrained Alexnet and Googlenet, application codes for the Alexnet-Googlenet-SVM hybrid CNN method are written in Matlab R2019a.

In order to demonstrate the success of the proposed model, WBC images were first classified with pretrained models, Alexnet and Googlenet. Secondly, the images are classified with the Alexnet-Googlenet-SVM model. To test the performance of the proposed approach, Sensitivity, Accuracy, Precision, F1-Score, AUC parameters are used. Table 1 gives the confusion matrix parameter definitions and metrics.

Firstly, classification was made with pretrained Alexnet and Googlenet architecture. Table 2 shows the values of performance parameters obtained with Alexnet and Googlenet for two data sets. In the classification made with Alexnet for Kaggle data set, 30 images from eosinophil class, 41 from Neutrophil class and 2 from Monocytle class, 73 images in total were misclassified. Overal accuracy $92.64 \%$ was achieved. Overall accuracy $95.74 \%$ was obtained with Googlenet, where relatively better accuracy results were obtained. With this architecture, 32 images from the Eosinophil class, 1 from the Lynphocyte class and 5 from the Monocytle class, 38 images in total were misclassified. When the results obtained for the LISC data set are examined, overall accuracy with Googlenet is $96.47 \%$. A total of 14 images from the Eonishopil class and 2 from the Lymphocyte class and 11 from the Monocyte class were classified incorrectly. In the classification made with Alexnet, 13 images from the Eonishopil class and 3 from the Lymphocyte class and 1 from the Monocyte class were

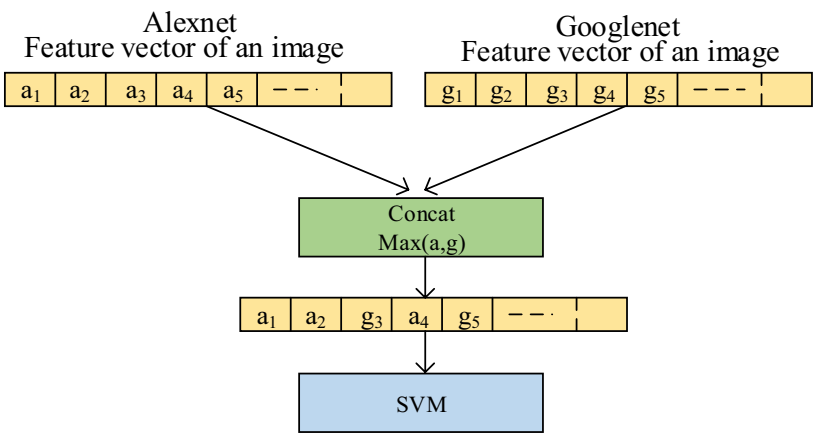

Fig. 4 Concat function 
Table 1 Confusion matrix parameters and metrics

\begin{tabular}{|c|c|c|c|c|c|c|}
\hline \multirow{7}{*}{ 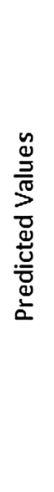 } & \multirow[b]{3}{*}{ Positive (1) } & \multicolumn{2}{|c|}{ Actual Values } & True Positives (TP): predicting patients as patients & Accuracy & $A=\frac{T P+T N}{T P+T N+F P+F N}$ \\
\hline & & Positive (1) & Negative (0) & & & \\
\hline & & TP & FP & & & \\
\hline & Negative (0) & $\mathrm{FN}$ & TN & & & \\
\hline & & & & True Negatives (TN): predicting non-patient as non-patient & Sensitivity & $S=\frac{T P}{T P+F N}$ \\
\hline & & & & False Positives (FP): predicting non-patients as patients & Precision & $P=\frac{T P}{T P+D}$ \\
\hline & & & & False Negatives (FN): predicting patient people as not-patient & $\mathrm{F}_{1}$-Score & $F_{1}=\frac{2 T P}{2 T P+F N+F P}$ \\
\hline
\end{tabular}

Table 2 Pretrained Alexnet and Googlenet performance parameters

\begin{tabular}{|c|c|c|c|c|c|c|}
\hline Type & Truth & Classified & $A(\%)$ & $\mathrm{P}(\%)$ & $\mathrm{S}(\%)$ & F1 (\%) \\
\hline \multicolumn{7}{|c|}{ Kaggle data set } \\
\hline \multicolumn{7}{|c|}{ Alexnet } \\
\hline$E$ & 230 & 248 & 95.77 & 88.0 & 95.0 & 0.91 \\
\hline L & 251 & 248 & 99.7 & 100.0 & 99.0 & 0.99 \\
\hline M & 278 & 248 & 96.57 & 99.0 & 88.0 & 0.94 \\
\hline $\mathrm{N}$ & 233 & 248 & 93.25 & 83.0 & 89.0 & 0.86 \\
\hline \multicolumn{7}{|c|}{ Googlenet } \\
\hline$E$ & 216 & 248 & 96.77 & 87.0 & 100.0 & 0.93 \\
\hline L & 247 & 248 & 99.9 & 100.0 & 100.0 & 1.0 \\
\hline M & 243 & 248 & 99.5 & 98.0 & 100.0 & 0.99 \\
\hline $\mathrm{N}$ & 286 & 248 & 96.17 & 100 & 87.0 & 0.93 \\
\hline \multicolumn{7}{|c|}{ LISC data set } \\
\hline \multicolumn{7}{|c|}{ Alexnet } \\
\hline$E$ & 90 & 99 & 97.73 & 91.0 & 100.0 & 0.95 \\
\hline L & 97 & 99 & 98.99 & 97.0 & 99.0 & 0.98 \\
\hline M & 109 & 99 & 96.97 & 99.0 & 90.0 & 0.94 \\
\hline $\mathrm{N}$ & 100 & 99 & 99.75 & 100.0 & 99.0 & 0.99 \\
\hline \multicolumn{7}{|c|}{ Googlenet } \\
\hline$E$ & 98 & 99 & 99.75 & 99.0 & 100.0 & 0.99 \\
\hline L & 108 & 99 & 96.73 & 98.0 & 90.0 & 0.94 \\
\hline M & 91 & 99 & 96.47 & 89.0 & 97.0 & 0.93 \\
\hline $\mathrm{N}$ & 99 & 99 & 100.0 & 100.0 & 100.0 & 1.0 \\
\hline
\end{tabular}

classified incorrectly. Overall accuracy is $96.72 \%$. According to Table 2, similar results were obtained for both data sets with Alexnet and Googlenet.

According to Table 2, considering the overall accuracy and $\mathrm{F} 1$ score, the results are likely to be improved. With this target, WBC images are classified with the Alexnet-Googlenet-SVM hybrid CNN model. The biggest factor in the accuracy of the model, Alexnetin Pool5, is the Concat layer that combines the feature vectors in the Avg_pool layers of the Google. For the feature vector to be obtained in the Concat layer, the maximum, minimum and average functions are applied to the feature vectors in the Pool5 and
Avg_pool layers. The feature vectors obtained are given as an input to the SVM algorithm. SVM places the attributes from each data image on the coordinate plane. Then the classification is done by finding the hyper-plane that best separates the classes. Classification is made by taking $\mathrm{k}$-fold cros validation 10 in SVM algorithm. The highest performance was achieved with the maximum function. Table 3 shows the classification performance parameters obtained with the Alexnet-Googlenet-SVM model for both data sets.

For the Kaggle data set, 2 images from the Eosinophil class and 1 from the Neutrophil class were misclassified. 
Table 3 Alexnet-GooglenetSVM classification results

\begin{tabular}{lclllll}
\hline Type & Truth & Classified & A (\%) & P (\%) & S (\%) & F1 \\
\hline \multicolumn{2}{l}{ Kaggle data set } & & & & & \\
$\mathrm{E}$ & 249 & 248 & 99.7 & 100.0 & 99.0 & 0.99 \\
$\mathrm{~L}$ & 248 & 248 & 100 & 100.0 & 100.0 & 1.0 \\
$\mathrm{M}$ & 248 & 248 & 100 & 100.0 & 100.0 & 1.0 \\
$\mathrm{~N}$ & 247 & 248 & 99.7 & 99.0 & 100.0 & 0.99 \\
LISC data set & & & & & & \\
$\mathrm{E}$ & 96 & 99 & 99.24 & 97.0 & 100.0 & 0.98 \\
$\mathrm{~L}$ & 97 & 99 & 98.99 & 97.0 & 99.0 & 0.98 \\
$\mathrm{M}$ & 104 & 99 & 98.23 & 99.0 & 94.0 & 0.97 \\
$\mathrm{~N}$ & 99 & 99 & 100.0 & 100.0 & 100.0 & 1.0 \\
\hline
\end{tabular}

E: Eosinophil, L:Lymphocyte, M:Monocyte, N:Neutrophil
For the LISC data set, 3 images from the Eosinophil class, 3 from the Lymphocyte class, and 1 image from the Monocyte class were misclassified. According to the data presented in Table 3, for the Kaggle data set, overall accuracy is $99.7 \%$ and $\mathrm{F} 1$ score is 0.99 and for the LISC data set overall accuracy is $98.23 \%$ is $\mathrm{F} 1$ score 0.98 . Another parameter used to determine the classification performance is the Area Value Under the ROC curve known as AUC. The size of the area under the curve indicates the accuracy and reliability of the classification model. Figure 5 shows the ROC curves obtained with the Alexnet-Gogglenet-SVM hybrid model for Kaggle data set. For the Eosinophil, Lymphocyte, Monocyte and Neutrophil class in the Kaggle data set, the AUC is $0.984,0.999,0.996$ and 0.984 , respectively.

Figure 6 shows the ROC curves obtained with the Alexnet-Gogglenet-SVM hybrid model for the LISC data set. For the Eosinophil, Monocyte, Lymphocyte and Neutrophil class in the LISC dataset, the AUC is $0.994,0.974$, 0.967 and 0.998 , respectively.

The proposed method successfully classifies according to the ROC curves drawn separately for each class and the performance parameter values obtained. To prove this success, a literature comparison is presented in Table 4.

Considering the results obtained for the Kaggle data set, in most of the classification metrics for each WBC class, our Hybrid CNN model has better value than other studies. Togaçar et al. and Kutlu et, with the highest classification accuracy using CNN models. get. The accuracy of the studies conducted by $99 \%$. When the results obtained with the Alexnet-Googlenet-SVM model were compared with the mentioned studies, more successful results were obtained in terms of accuracy and F1. Accuracy for each class is over $99 \%$. The overall classification performance of our hybrid CNN model, especially in terms of F1 score and AUC, has shown that it is better than other CNN models, which provides reliability to our proposed hybrid CNN model. According to the results of the LISC dataset, the accuracy is greater than $98 \%$ for all WBC classes. The performance parameters obtained for this data set are in parallel with the studies in the literature.

\section{Conclusion}

The WBC test provides information about the amount of white blood cells in the blood. If the number of white blood cells is outside the normal range, this leads to the occurrence of various diseases. In this article, Eosinophil, Lymphocyte, Monocyte, and Neutrophil WBC images in 2 different data sets are classified by the Alexnet-GooglenetSVM hybrid CNN method. There are two important reasons behind the success of our proposed methods. The first is to evaluate Alexnet's pool5, Googlenet's feature vector in the Avg_pool layer. For this, the feature with the maximum value from two feature vectors was used for classification. The second is the use of SVM, a powerful classification algorithm. Considering both the literature and the results obtained, we can also claim that our Hybrid CNN model can be used for the application of medical diagnostic systems. 


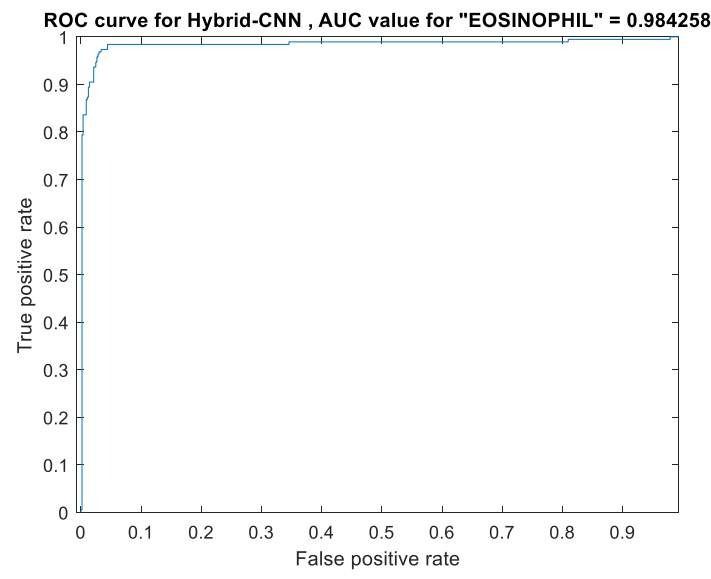

(a) Eosinophil

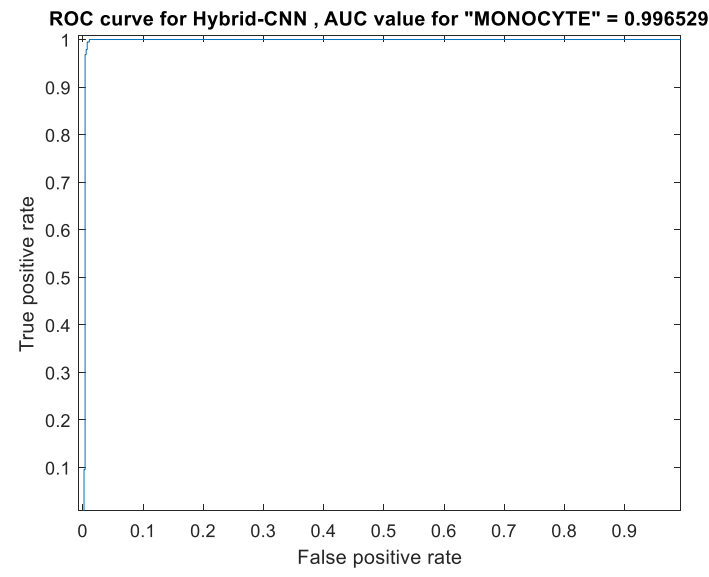

(c) Monocyte

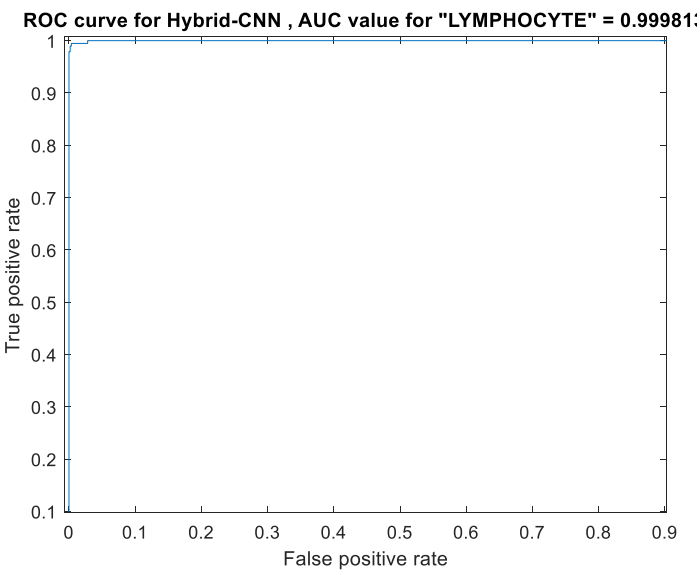

(b) Lymphocyte

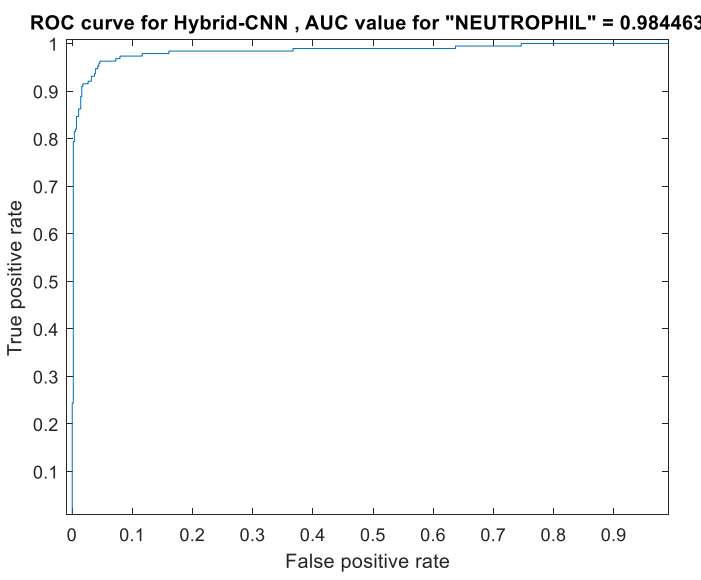

(d) Neutrophil

Fig. 5 Eosinophil, lymphocyte, monocyte and neutrophil ROC curves for Kaggle data set 


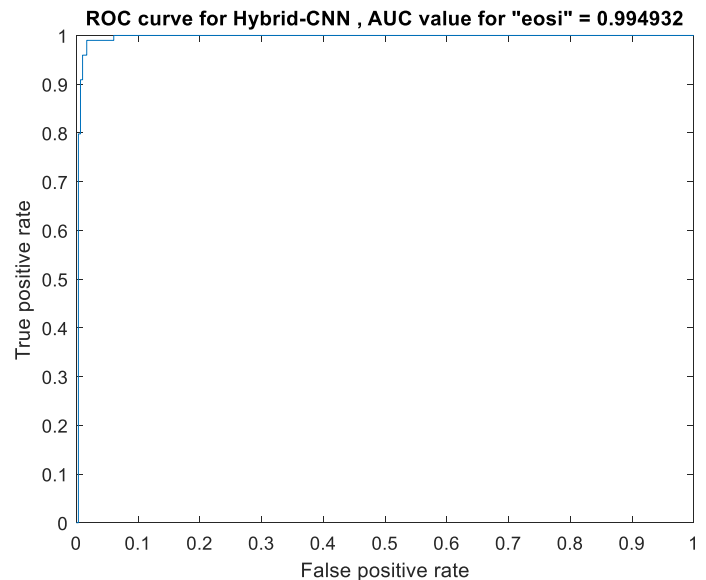

(a) Eosinophil

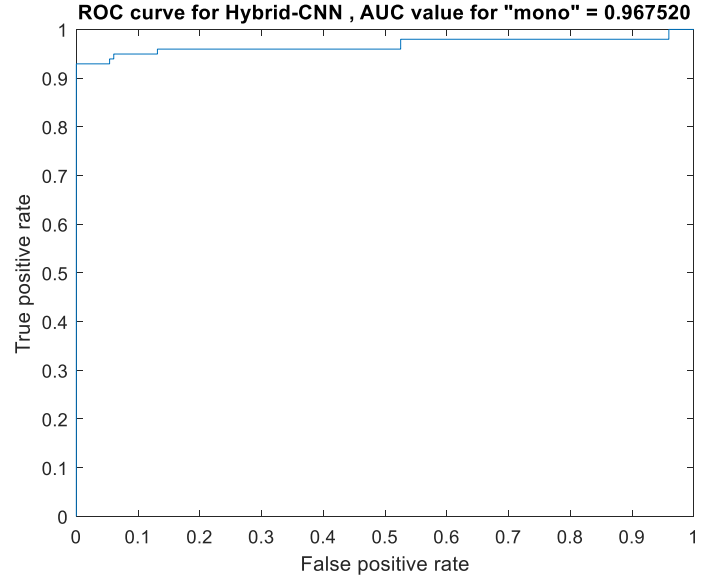

(c) Monocyte

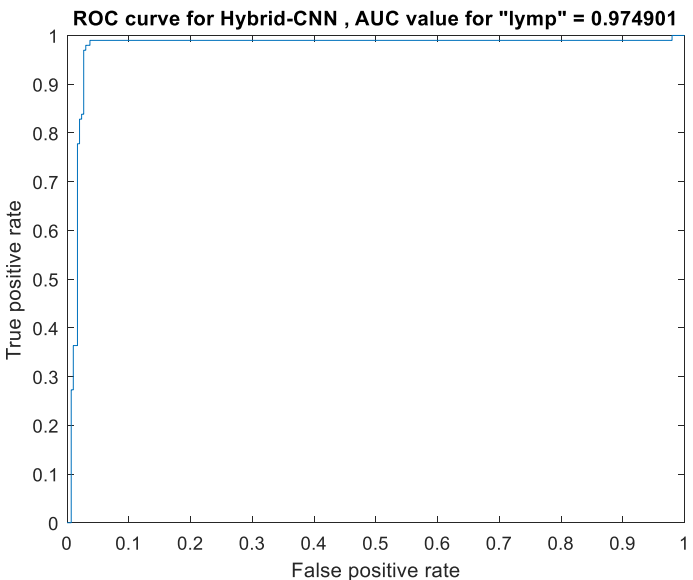

(b) Lymphocyte

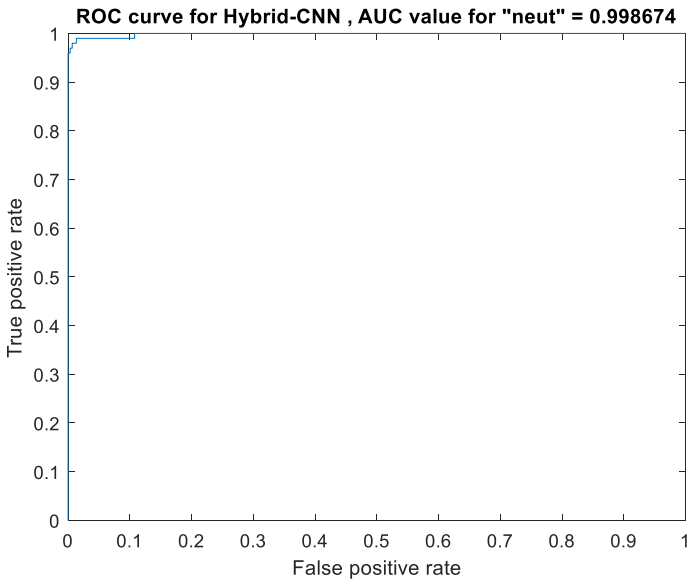

(d) Neutrophil

Fig. 6 Eosinophil, lymphocyte, monocyte and neutrophil ROC curves for LISC data set 
Table 4 Comparison of the proposed method with the literature

\begin{tabular}{|c|c|c|c|c|c|c|}
\hline Reference & WBC Type & $A(\%)$ & $\mathrm{P}(\%)$ & $\mathrm{S}(\%)$ & F1 & Method \\
\hline \multirow[t]{4}{*}{ [1] } & Eosinophil & 96.24 & 93.0 & 97.86 & 0.92 & \multirow[t]{4}{*}{ CNN-RNN } \\
\hline & Lymphocyte & 99.92 & 100.0 & 99.94 & 1.0 & \\
\hline & Monocyte & 94.44 & 96.0 & 98.96 & 0.88 & \\
\hline & Neutrophil & 91.08 & 78.0 & 90.88 & 0.76 & \\
\hline \multirow[t]{4}{*}{ [2] } & Eosinophil & 94.80 & 94.0 & 98.28 & 0.89 & \multirow[t]{4}{*}{ Fused CNN } \\
\hline & Lymphocyte & 100.0 & 100 & 100.0 & 1.0 & \\
\hline & Monocyte & 93.86 & 100 & 100.0 & 0.86 & \\
\hline & Neutrophil & 88.66 & 71.0 & 86.37 & 0.82 & \\
\hline \multirow[t]{4}{*}{ [3] } & Eosinophil & 88.19 & 78.0 & 93.18 & 0.75 & \multirow[t]{4}{*}{ Deep Neural Network } \\
\hline & Lymphocyte & 99.92 & 94.0 & 97.42 & 0.95 & \\
\hline & Monocyte & 88.42 & 86.0 & 88.42 & 0.73 & \\
\hline & Neutrophil & 85.53 & 68.0 & 90.55 & 0.84 & \\
\hline \multirow[t]{4}{*}{ [4] } & Eosinophil & 95.05 & 96.0 & 98.87 & 0.89 & \multirow[t]{4}{*}{ Fused CNN } \\
\hline & Lymphocyte & 99.84 & 99.0 & 99.78 & 1.0 & \\
\hline & Monocyte & 95.33 & 100.0 & 100.0 & 0.9 & \\
\hline & Neutrophil & 95.93 & 93.0 & 97.5 & 0.92 & \\
\hline \multirow[t]{4}{*}{ [5] } & Eosinophil & 96.48 & 93.0 & 97.50 & 0.93 & \multirow[t]{4}{*}{ CNN } \\
\hline & Lymphocyte & 99.92 & 100.0 & 99.89 & 1.00 & \\
\hline & Monocyte & 99.45 & 99.0 & 99.58 & 0.99 & \\
\hline & Neutrophil & 95.93 & 93.0 & 97.50 & 0.92 & \\
\hline \multirow[t]{4}{*}{ [10] } & Eosinophil & 96.16 & 90.82 & 83.20 & - & \multirow[t]{4}{*}{ Resnet50 } \\
\hline & Lymphocyte & 99.52 & 96.0 & 97.16 & & \\
\hline & Monocyte & 98.40 & 986.8 & 90.40 & & \\
\hline & Neutrophil & 95.04 & 91.07 & 82.59 & & \\
\hline \multirow[t]{4}{*}{ [18] } & Eosinophil & 96.34 & 94.92 & 90.86 & - & \multirow[t]{4}{*}{ Alexnet-QDA } \\
\hline & Lymphocyte & 99.45 & 97.99 & 99.80 & & \\
\hline & Monocyte & 98.96 & 95.83 & 100.0 & & \\
\hline & Neutrophil & 96.37 & 93.49 & 92.09 & & \\
\hline \multirow[t]{4}{*}{ [19] } & Eosinophil & 99.3 & - & 93.5 & - & \multirow[t]{4}{*}{ Neural Network } \\
\hline & Lymphocyte & 100.0 & & 100.0 & & \\
\hline & Monocyte & 99.8 & & 99.7 & & \\
\hline & Neutrophil & 99.7 & & 100.0 & & \\
\hline \multirow[t]{4}{*}{ Proposed model (For Kaggle) } & Eosinophil & 99.7 & 100.0 & 99.0 & 0.99 & \multirow[t]{4}{*}{ Alexnet-Googlenet-SVM } \\
\hline & Lymphocyte & 100.0 & 100.0 & 100.0 & 1.0 & \\
\hline & Monocyte & 100.0 & 100.0 & 100.0 & 1.0 & \\
\hline & Neutrophil & 99.7 & 99.0 & 100.0 & 0.99 & \\
\hline \multirow[t]{4}{*}{ Proposed model (For LISC) } & Eosinophil & 99.24 & 97.0 & 100.0 & 0.98 & \multirow[t]{4}{*}{ Alexnet-Googlenet-SVM } \\
\hline & Lymphocyte & 98.99 & 97.0 & 99.0 & 0.98 & \\
\hline & Monocyte & 98.23 & 99.0 & 94.0 & 0.97 & \\
\hline & Neutrophil & 100.0 & 100.0 & 100.0 & 1.0 & \\
\hline
\end{tabular}

\section{Declarations}

Conflict of interest The authors declare that there are no conflicts of interest.

Open Access This article is licensed under a Creative Commons Attribution 4.0 International License, which permits use, sharing, adaptation, distribution and reproduction in any medium or format, as long as you give appropriate credit to the original author(s) and the source, provide a link to the Creative Commons licence, and indicate if changes were made. The images or other third party material in this article are included in the article's Creative Commons licence, unless indicated otherwise in a credit line to the material. If material is not included in the article's Creative Commons licence and your intended use is not permitted by statutory regulation or exceeds the permitted use, you will need to obtain permission directly from the copyright holder. To view a copy of this licence, visit http://creativecommons. org/licenses/by/4.0/. 


\section{References}

1. Liang $\mathrm{G}$, Hong $\mathrm{H}$, Xie W, Zheng L (2018) Combining convolutional neural network with recursive neural network for blood cell image classification. IEEE Access 6:36188-36197

2. Pang S, Du A, Orgun MA, Yu Z (2019) A novel fused convolutional neural network for biomedical image classification. Med Biol Eng Comput 57(1):107-121

3. Yu W, Chang J, Yang C, Zhang L, Shen H, Xia Y, Sha J (2017) Automatic classification of leukocytes using deep neural network. In: IEEE 12th international conference on ASIC (ASICON), pp 1041-1044

4. Banik PP, Saha R, Kim K-D (2019)Fused convolutional neural network for white blood cell image classification. In: International conference on artificial Intelligence in information and communication

5. Banik PP, Saha R, Kim K-D (2020) An automatic nucleus segmentation and CNN model based classification method of white blood cell. Exp Syst Appl 149:113211

6. Macawile MJ, Quiñones VV, Ballado A, Cruz JD, Caya MV (2018) White blood cell classification and counting using convolutional neural network. In: 2018 3rd International conference on control and robotics engineering (ICCRE), Nagoya, pp 259-263

7. Sahlol AT, Kollmannsberger P, Ewees AA (2020) Efficient classification of white blood cell leukemia with ımproved swarm optimization of deep features. Sci Rep 10: 2536

8. Ramesh N, Dangott B, Salama ME, Tasdizen T (2012) Isolation and two-step classification of normal white blood cells in peripheral blood smears. J Pathol Inf 3:13

9. Su MC, Cheng CY, Wang PC (2014) A neural-network-based approach to white blood cell classification. Sci World J 2014:796371

10. Kutlu H, Avci E, Özyurt F (2020) White blood cells detection and classification based on regional convolutional neural networks. Med Hypotheses 135:109472

11. Barrero CMR, Gabalan R, Alberto L, Roa Guerrero EE (2018) A novel approach for objective assessment of white blood cells using computational vision algorithms. Adv Hematol 2018:4716370

12. Habibzadeh M, Krzyżak A, Fevens T (2013) Comparative study of shape intensity and texture features and support vector machine for white blood cell classification. J Theoret Appl Comput Sci 7(1):20-35

13. Zhao J, Zhang M, Zhou Z, Chu J, Cao F (2017) Automatic detection and classification of leukocytes using convolutional neural networks. Med Biol Eng Comput 55:1287-1301

14. Di Ruberto C, Loddo A, Puglisi G (2020) Blob detection and deep learning for leukemic blood image analysis. Appl Sci 10:1176

15. Baydilli YY, Atila Ü (2020) Classification of white blood cells using capsule networks. Comput Med Imaging Graph 80:101699
16. Deepak G, Jatin A, Utkarsh A, Khanna A, Albuquerque VHC (2019) Optimized binary bat algorithm for classification of white blood cells. Measurement, 143

17. Shahin Al, Guo Y, Amin KM, Sharawi AA (2017) White blood cells Identification system based on convolutional deep neural learning networks. Comput Methods Programs Biomed 168:69-80

18. Togacar M, Ergen B, Sertkaya ME (2019) Subclass separation of white blood cell images using convolutional neural network models. Elektron Ir Elektrotechnika 25:63-68

19. Hegde RB, Prasad K, Hebbar H, Singh BMK (2019) Comparison of traditional image processing and deep learning approaches for classification of white blood cells in peripheral blood smear images. Biocybern Biomed Eng 39(2):382-392

20. Malkawi A, Al-Assi A, Salameh R, Alquran T, Alqudah H (2020) White blood cells classification using convolutional neural network hybrid system. In 2020 IEEE 5th middle east and Africa conference on biomedical engineering (MECBME), pp $1-5$

21. Rezatofighi SH, Soltanian-Zadeh H (2011) Automatic recognition of five types of white blood cells in peripheral blood. Comput Med Imaging Graph 35(4):333-343

22. Prinyakupt J, Pluempitiwiriyawej C (2015) Segmentation of white blood cells and comparison of cell morphology by linear and naïveBayes classifiers. BioMed Eng 14:63

23. Sarrafzadeh O, Rabbani H, Talebi A, Banaem H (2014) Selection of the best features for leukocytes classification in blood smear microscopic images. Med Imaging Dig Pathol 9041:1-8

24. Alqudah AM, Al-Ta'ani O, Al-Badarneh A (2018) Automatic segmentation and classification of white blood cells in peripheral blood samples. J Eng Sci Technol Rev 11(6):7-13

25. https://www.kaggle.com/paultimothymooney/blood-cells, Accessed 01.04.2020.

26. http://users.cecs.anu.edu.au/ hrezatofighi/Data/Leukocyte\%20Data.htm, Accessed 01.04.2020.

27. A. Krizhevsky, I. Sutskever, G. Hinton, "ImageNet Classification with Deep Convolutional Neural Networks", Proc. Neural Information and Processing Systems, 2012

28. ImageNet. http://www.image-net.org

29. Kassem MA, Hosny KM, Foaud MM (2020) Skin melanoma classification using $\mathrm{ROI}$ and data augmentation with deep convolutional neural networks. Multimed Tools Appl 79(33):24029-24055

30. Çınar A, Tuncer SA (2021) Classification of normal sinus rhythm, abnormal arrhythmia and congestive heart failure ECG signals using LSTM and hybrid CNN-SVM deep neural networks. Comput Methods Biomech Biomed Eng. https:// doi.org/10.1080/10255842.2020.1821192

31. Kassem MA, Hosny KM, Fouad MM (2020) Skin lesions classification into eight classes for ISIC 2019 using deep convolutional neural network and transfer learning. IEEE Access 8:114822-114832

Publisher's Note Springer Nature remains neutral with regard to jurisdictional claims in published maps and institutional affiliations. 